\title{
Measurement of antenna surfaces from in- and out-of-focus beam maps using astronomical sources
}

\begin{abstract}
B. Nikolic, R. E. Hills, and J. S. Richer
Mullard Radio Astronomy Observatory, Cavendish Laboratory, Cambridge CB3 OHE, UK

e-mail: bnikolic@nrao.edu

Received 14 May 2006 / Accepted 4 January 2007

ABSTRACT

We present a technique for the accurate estimation of large-scale errors in an antenna surface using astronomical sources and detectors. The technique requires several out-of-focus images of a compact source and the signal-to-noise ratio needs to be good but not unreasonably high. For a given pattern of surface errors, the expected form of such images can be calculated directly. We show that it is possible to solve the inverse problem of finding the surface errors from the images in a stable manner using standard numerical techniques. To do this we describe the surface error as a linear combination of a suitable set of basis functions (we use Zernike polynomials). We present simulations illustrating the technique and in particular we investigate the effects of receiver noise and pointing errors. Measurements of the 15-m James Clerk Maxwell telescope made using this technique are presented as an example. The key result is that good measurements of errors on large spatial scales can be obtained if the input images have a signal-to-noise ratio of order 100 or more. The important advantage of this technique over transmitter-based holography is that it allows measurements at arbitrary elevation angles, so allowing one to characterise the large scale deformations in an antenna as a function of elevation.
\end{abstract}

Key words. telescopes

\section{Introduction}

Measuring the shape and deformations of radio antennas using microwaves (often called holography) is useful both for correcting the surface errors directly, and for testing theoretical models of the behaviour of telescope structures. Both of those are in turn invaluable for the characterisation of current and future generations of high-precision 10-20 m class antennas which are required to have high gain at frequencies up to and beyond $1 \mathrm{THz}$, and of course for larger antennas operating at lower frequencies. Maximising the surface accuracy of existing and new antennas is of increasing scientific importance for two reasons: it increases the gain (particularly at higher frequencies), so allowing detection of fainter compact sources, and it reduces the amount of power in the "error beam", so allowing imaging of extended sources with higher dynamic range.

In the phase-retrieval approach to millimetre-wave holography, only the power pattern of the antenna is measured, usually at two or more different focus settings. The phase of the signal in the aperture is later recovered by numerical processing (Morris 1985; Anderson \& Sali 1985). This technique has been applied with considerable success on a number of large antennas, but usually only with artificial sources, i.e. transmitters on the ground or on spacecraft (e.g., Fuhr et al. 1993). This is typically possible only at a small range of elevations: for ground based beacons, for example, measurements are essentially only possible with the telescope pointed within ten degrees or so of the horizon, and this does not allow measurement of gravitation deformations of the surface.

This paper describes the development of a technique for measuring surface errors with moderate spatial resolution by observing astronomical sources, using existing astronomical receivers on the telescopes. The new approach uses numerical fitting of a parameterised description of the surface errors, and of the amplitude of the receiver's illumination pattern. The technique is flexible. It can be adapted straightforwardly to various different observational techniques, including total power observations, and the various differencing schemes which involve movement of the secondary reflector ("beam switching") and/or primary reflector ("nodding"). Extended sources, such as planets, can also be accommodated. In future, the increasing availability of astronomical array detectors will make the mapping process particularly quick and efficient.

\section{The technique}

If both the amplitude and the phase of the far-field beam pattern of a telescope are measured, a simple Fourier inversion will give the aperture function and thus the deformations of the telescope. This is the conventional with-phase holography which is widely used for measurement of radio-telescopes either when they already operate as parts of interferometers (Scott \& Ryle 1977) or when equipped with an auxiliary phase-reference antenna (e.g., Morris et al. 1988). Measuring just the power pattern of the infocus beam under-determines the aperture function - for example, reversing the sign of surface errors produces no change in the power pattern. This degeneracy can be broken, and, additionally, partially independent data sets obtained, by introducing a known phase change across the aperture, for example by defocussing the telescope by a known amount. It is also true that small errors in the surface of the reflector have a much greater effect on defocussed images than on the in-focus one. This is the main reason why the method described here works well with only moderately high signal to noise ratio.

Due to the non-linearity of the measurement process, a straightforward inversion from the observed beam power 
patterns to the aperture plane phase distribution is not possible. Rather, the aperture needs to be parameterised in terms of a suitable set of basis functions and a numerical fitting procedure employed.

We parameterise the aperture phase distribution - which is determined by the combination of surface errors, phase response of the receiver and any mis-collimation of the telescope - in terms of a fixed number of coefficients of Zernike circle polynomial functions, i.e.:

$\phi(x, y)=\sum_{n=1}^{n_{\max }} \sum_{l=-n,-n+2, \ldots, n} a_{n, l} Z_{n, l}(x, y)$,

where $\phi(x, y)$ is the aperture phase distribution, $a_{n, l}$ are the coefficients to be determined and $Z_{n, l}(x, y)$ are the Zernike circle polynomials labeled with to their radial $(n)$ and angular $(l)$ orders (see, for example, Born \& Wolf 1970, for a definition). For each radial order $n$ there the are $n+1$ possible angular orders, and so the parameter $n_{\max }$ determines the total number of coefficients $[(n+1)(n+2) / 2]$ which are to be found by fitting.

For example, if the maximum radial order is six (approximately the value typically used in practice, see later and Nikolic et al. 2007), there are a total of 28 coefficients to be found. The smallest scales present in the highest order $(n=6)$ functions correspond to scales of roughly one fifth the dish diameter.

This choice of parametrisation in terms of Zernike polynomials has several advantages, in particular that they are orthogonal on the unit circle. This strict orthogonality breaks down when applied to practical measurements of radio antennas because the illumination is not uniform across the aperture, due to the edge taper and central blockage. Nonetheless, approximate orthogonality is maintained. Further, by restricting the highest order of Zernike polynomial used, the results can be made less sensitive to the poorly-constrained small-scale errors on the surface. Finally, some of the low-order Zernike polynomials correspond closely to aberrations which are likely to be present in the optics. For example, a misalignment of the secondary reflector in a Cassegrain telescope away from the optical axis gives rise to a aberration which is closely approximated by the $n=3$, $l= \pm 1$ Zernike polynomials.

To model the far-field antenna power pattern, besides the phase of the aperture function, it is also necessary to know its amplitude [often referred to as the illumination, $I(x, y)$, of the primary reflector]. This is determined in practice by the properties of the receiving system which couples detectors to the primary antenna surface. When these are known with sufficient accuracy, the amplitude distribution of the aperture function can be determined a priori and taken as a given in the model. We have however found that it is generally better to model the amplitude as a function of a small number of free parameters and solve for these simultaneously with the coefficients $a_{n, l}$ that determine the phase. We use a two-dimensional Gaussian as a model for the amplitude with free parameters describing its width, offset with respect to the axis, etc., i.e.:

$I(x, y)=I_{0} \exp \left[-\frac{\left(x-x_{0}\right)^{2}+\left(y-y_{0}\right)^{2}}{R^{2}} \sigma_{r}\right]$,

where $R$ is the radius of the primary reflector, $\left(x_{0}, y_{0}\right)$ define the centre of the illumination on the primary reflector and $\sigma_{r}$ is the illumination taper.

If the best-fitting model amplitude is not in agreement with the expected amplitude distribution, then this may indicate a problem in the way the data have been processed or a problem in the receiving system.

\subsection{Phase change due to defocus}

For a Cassegrain telescope, in the ray-tracing approximation, the extra path $\delta$ due to a radial defocus of the telescope by a distance $d_{Z}$ is given by:

$\delta\left(x, y ; d_{Z}\right)=d_{Z}\left(\frac{1-a^{2}}{1+a^{2}}+\frac{1-b^{2}}{1+b^{2}}\right)$

where $a=r /(2 f), b=r /(2 F), r=\sqrt{\left(x^{2}+y^{2}\right)}$ is the radius in the aperture plane from the optical axis of the telescope, $f$ is the focal length of the primary reflector, $F$ is the effective total focal length of the telescope at the Cassegrain focus, and a positive value of $d_{Z}$ corresponds to a movement of the secondary reflector away from the primary.

Putting all of the above together, the aperture function $A(x, y)$ may be written as:

$A(x, y)=\Theta\left(R^{2}-x^{2}-y^{2}\right) I(x, y) \exp \left[\phi(x, y)+\delta\left(x, y ; d_{Z}\right)\right]$

where $\Theta$ is the Heaviside step function which described the truncation of the aperture function at the edge of the primary reflector.

\subsection{The fitting procedure}

The parametrised form of the aperture function (Eq. (4)) may be easily computed on a convenient rectangular grid making it straightforward to calculate the corresponding power beam pattern using the fast Fourier transform. The expected out-of-focus beam patterns are computed by adding to the aperture function, prior to the Fourier transform, the additional phase change due to the defocus as given by Eq. (3) for the case of a Cassegrain telescope. If the measurement was not of the true antenna power pattern - as may be case if a differencing technique is used, or if the source employed was not sufficiently point-like - this can be taken into account by an appropriate convolution of the model power-pattern, in either the sky or the Fourier domain.

In the procedure we use, the model power beam pattern is compared to observed data (labeled $\boldsymbol{D}$ ) by interpolating the model produced using the fast Fourier Transform to the position of each observed data point (producing the model vector $\boldsymbol{y}$ ), eliminating the need for the observed data to be on a regular grid or for it to be re-gridded in any way. This is desirable since interpolation and regridding of the observed data generally causes a loss of information whereas interpolating the model does not. The computational burden of this step is reduced by storing the interpolation coefficients between iterations.

The agreement between a model and observed data is characterised by the vector of residuals, $r_{i}$, weighted by an estimate of the measurement error, $\sigma_{i}$, at each data point; i.e.,:

$r_{i}=\frac{D_{i}-y_{i}}{\sigma_{i}}$

In the simplest case, the measurement errors will be dominated by thermal noise characterised by the system temperature, the bandwidth used and the integration time. In practice, other factors can significantly contribute to measurement errors. These may include imperfect removal of a time-varying atmospheric emission, variation in the gain of the receiver, source noise (in the case of observations of very strong narrow line sources, such as $\mathrm{SiO}$ masers), pointing errors of the telescope and radio "seeing" (direction of arrival fluctuations due to the atmosphere).

The best fitting telescope surface is found by minimising the magnitude of the error-weighted residual vector, i.e., $|r|$. At this 
point a "prior" on the surface deformations, or equivalently, on the phase of the aperture function, may be added to the minimisation process as an extra residual. For example one can add to this quantity the value of the root-mean-square of the surface error in the model, multiplied by a constant, do the minimisation and then inspect the resulting match between the model beams and the data as the value of this constant is varied. A large value of the constant favours solutions with small surface errors, so by gradually reducing it one can choose the most conservative solution which provides an adequate fit. This is helpful if, for example, one is using the measurements to make adjustments of the surface.

Our initial investigations employed the downhill-simplex minimiser, and we found that for small to moderate phase errors (i.e. less than a whole turn of phase peak-to-peak) the posterior distribution is in general convex and with a well defined global maximum. This means that it should be sufficient to do a simple search for the maximum of the posterior distribution rather than characterise it in full. We therefore routinely employ the relatively efficient Levenberg-Marquardt algorithm (Meyerdierks et al. 1997).

\section{Simulations}

The feasibility and accuracy, and the optimum observational approach, of the technique just described has been investigated using numerical simulations. These simulations have been carried out for a Cassegrain antenna with parameters similar to the proposed Atacama Large Millimetre Array (ALMA) antennas, i.e., with a primary reflector of $12 \mathrm{~m}$ diameter, effective focal lengths of $4.8 \mathrm{~m}$ and $96 \mathrm{~m}$ at primary and Cassegrain foci respectively, and a Gaussian illumination with a $12 \mathrm{~dB}$ edge taper. The main results can be straightforwardly reinterpreted for different antennas. The wavelength of simulated observations was $1 \mathrm{~mm}$.

The approach taken was to calculate theoretical beam maps, both in- and out-of-focus, for a perfect dish and to add to these beam-maps simulated noise and instrumental effects. These were then used as input to the fitting procedures.

The accuracy of the best-fitting surface is reported as $\epsilon$, the illumination-weighted root-mean-square of the phase of the aperture function. Since $\epsilon$ scales with wavelength, we express it in the of units of radians of phase, which at a given observing frequency are easily converted to a physical length. For example, at a typical observing frequency of $230 \mathrm{GHz}$ and assuming normal incidence (i.e., a large $f$-ratio aperture), a wavefront error of 0.1 radian corresponds to a deformation of the surface of $10.4 \mu \mathrm{m}$.

The fractional decrease of the on-axis antenna gain due to these errors is given approximately by $\epsilon^{2}$ (Ruze 1966), so the results of the simulations can be interpreted as follows. If the telescope is dominated by large scale errors, then after resetting a surface with an out-of-focus holography solution with $\epsilon=0.1$, then the efficiency of the telescope at the frequency at which the out-of-focus beam maps were made would be $\approx 99 \%$. The efficiency at three times that frequency will, however, be only $\approx 91 \%$.

The results of these simulations are strictly only applicable to antennas with surface errors much smaller than the observing wavelength. For antennas with significant deformations the errors on the measured surface may be expected to be somewhat larger. If, however, an iterative approach to correcting and re-measuring the antenna is taken, then it should be possible to approach the accuracies which are given below.

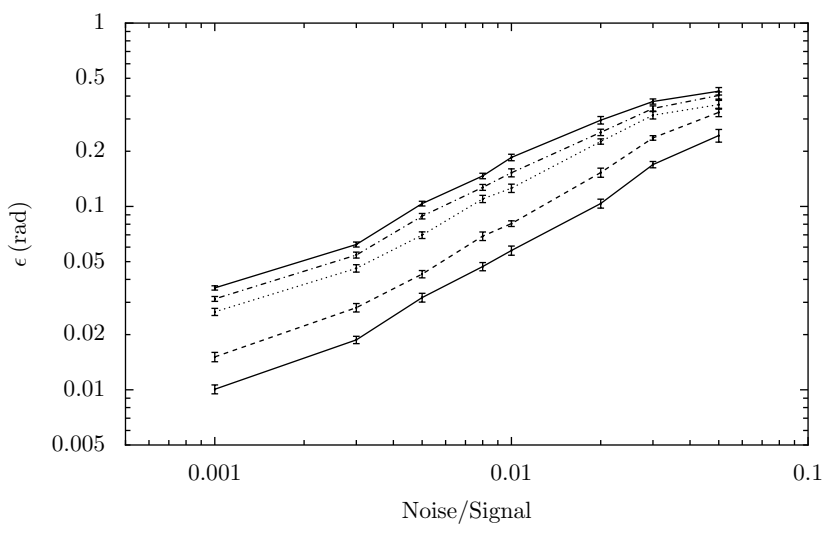

Fig. 1. The weighted root-mean-square error $(\epsilon$, in the units of radians of phase) of the best-fitting aperture phase as a function of peak signal to noise. The five traces correspond, bottom to top, to derived errors when fitting for coefficients of Zernike polynomials up to 4th, 5th, 6th, 7th and 8 th radial order. The out-of-focus maps were simulated at $\pm 2 \mathrm{~mm}$ defocus.

\subsection{Effect of noise in input maps}

We first consider the relationship between errors on the derived surface, the signal to noise ratio and the number of coefficients of Zernike polynomials being fitted for. A number of noisy realisations $\left(\boldsymbol{D}_{\mathrm{s}}^{\prime}\right)$ of the theoretical beams $\left(\boldsymbol{D}_{\mathrm{s}}\right)$ were derived using:

$\boldsymbol{D}_{\mathrm{s}}^{\prime}=\boldsymbol{D}_{\mathrm{s}}(1+\boldsymbol{m})+\boldsymbol{a}$

where $\boldsymbol{m}$ and $\boldsymbol{a}$ are random Gaussian variables with zero mean such that $\left\langle\boldsymbol{m}^{2}\right\rangle^{\frac{1}{2}}=m$ and $\left\langle\boldsymbol{a}^{2}\right\rangle^{\frac{1}{2}}=a$. The multiplicative noise is then simply determined by $m$, while we set $a$ so that the peak signal-to-noise is given by $\max \left(\boldsymbol{D}_{\mathrm{s}}\right) / a$.

The effect of thermal noise is illustrated in Fig. 1, which shows the surface errors from a number of simulated data sets of different signal-to-noise ratios, all with multiplicative noise set to $m=0.05$ and consisting of three Nyquist-sampled maps, one in-focus and one at $-2 \mathrm{~mm}$ and $+2 \mathrm{~mm}$ defocus each. Derived surfaces were calculated separately for parameterisations with maximum radial orders of Zernike polynomials ranging from 4 to 8 , thereby quantifying the increase in measurement error with increasing spatial resolution.

The relationship between the degree of defocus used for the out-of-focus maps and the accuracy with which the aperture phase distribution can be recovered is illustrated in Fig. 2. Since the area over which power is spread increases with increasing defocus, it may be expected that the accuracy is a function of the signal to noise in the maps. For this reason, simulations with three signal to noise ratios are shown in the figure, indicating that at better signal to noise ratios, a somewhat larger defocus produces optimal results. For this case, however, with a significant multiplicative noise level, the magnitude of the defocus is not very critical.

\subsection{Effect of pointing errors}

We next consider the extent to which pointing jitter degrades the accuracy of the inferred aperture phase. Such jitter may be the result of servo tracking errors, radio seeing or rapidly varying deformations of the telescope structure. The simplest way to model such errors in the pointing is by assuming that each sample in the observed maps is displaced by a random distance $\delta \theta, \delta \phi$ from its true position, with these displacement being Gaussian random 


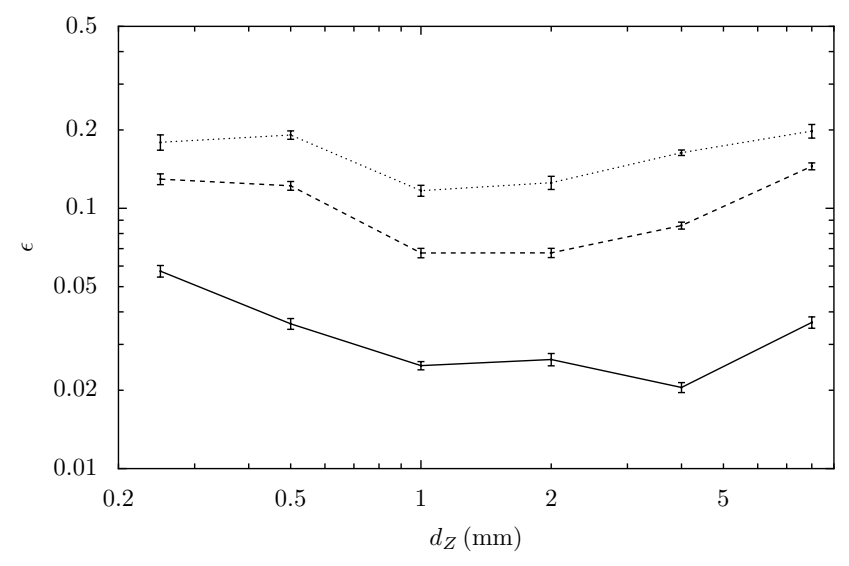

Fig. 2. The weighted root-mean-square error ( $\epsilon$, units radians of phase) of the best-fitting aperture phase as a function of the magnitude of defocus used in the out-of-focus maps for three noise to signal ratios (bottom to top ): $0.001,0.005$, and 0.01 . The maximum radial order of Zernike polynomials used in the fit was 6 and a multiplicative noise of 5 percent was added to the maps as before.

variables with variances of $\Delta^{2}$ and there being no correlation between samples.

Figure 3 shows the effect of such pointing errors in a set of simulated maps with a peak signal to noise ratio of 200 to 1 and 5 percent multiplicative noise. It can be seen that the accuracy of the technique rapidly gets worse as pointing errors become significant. For example, rms pointing jitter of 0.2 beam-widths increases the error in the derived surface by more then a factor of two when compared to the no-pointing jitter case.

We have also explored the impact on accuracy of the technique of using resolved sources for the beam maps. We find that for sources with sharp edges the accuracy is not much affected by their size. This means that objects like Venus and Mars are good sources for such measurements, but that Jupiter is somewhat less so because of limb darkening. We have even succeded in using Saturn when this was the only source available, but this did require a good model for the source.

\section{A sample measurement at the James Clerk Maxwell Telescope}

The James Clerk Maxwell Telescope is a 15-m submillimetre telescope with conventional Cassegrain optics, covering the atmospheric transmission bands from $150 \mathrm{GHz}$ to $1.5 \mathrm{THz}$ ( $2 \mathrm{~mm}$ to $200 \mu \mathrm{m}$ wavelength): its target surface accuracy is around $22 \mu \mathrm{m}$. We here present some sample out-of-focus holography measurements taken at the JCMT as an illustration of the technique.

These observations were made on Venus with a heterodyne receiver tuned to a frequency of $232.5 \mathrm{GHz}$, and utilising a chopper (with an 160 arcsec throw) for atmospheric rejection. The apparent diameter of Venus at the time of observations was 12 arcsec, smaller than the main beam of the telescope (which at this frequency has the full-width half-maximum of 20 arcsec), but large enough to require taking into account in the analysis. In total five maps were taken, although here we concentrate only on the in-focus and $\pm 2 \mathrm{~mm}$ out-of-focus maps. All of the maps were 320 arcsec long in the azimuth direction and 160 arcsec long in the elevation direction and were sampled at 8 arcsec intervals. They are shown in the top row of Fig. 4.

The large chop throw used in these observations means that it is necessary to take into account the aberrations introduced by

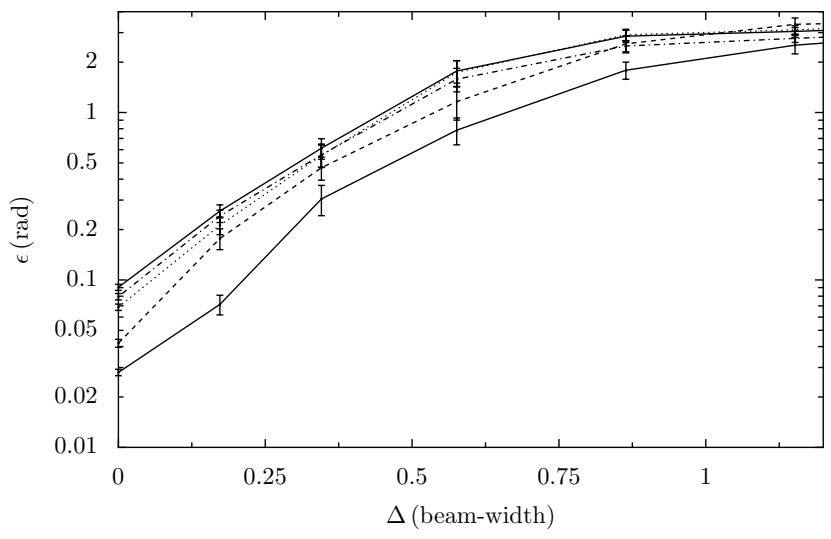

Fig. 3. Calculated surface error ( $\epsilon$, units radians of phase) as a function of $\Delta$, the root-mean-square of pointing errors, which are assumed to be uncorrelated between the samples. The traces correspond to, from bottom to top, to simulations with maximum Zernike radial orders of 4-8. The thermal signal to noise ratio is 200 to 1 with a five per-cent multiplicative noise as before.

the tilt of the secondary reflector which is required to deflect the beam. The phase change in the aperture plane corresponding to this aberration has been calculated using a ray-tracing software package: the dominant term is, as usually is the case, coma, with a magnitude of 0.072 radians at dish edge for a throw of 80 arcsec on the sky (i.e., the deflection from the centre beam for the throw used).

The best-fitting surface map is shown if Fig. 5 and the corresponding model beams in the bottom row of Fig. 4. These measurements were taken at a time when there was a known fault in the dish which resulted in an observed bump in one of the quadrants of the dish. They do not represent the current status of the JCMT surface.

Nikolic et al. (2007) present a detailed study of the Green Bank Telescope (a large millimetre-wave telescope), showing how gravitational and thermal effects can be measured and corrected using the technique. They also present a detailed practical verification of the technique.

\section{Discussion and conclusions}

A variation of the phase-retrieval approach to holography has been presented with the surface parametrised in terms of a basis set of continuous function in the aperture plane and with optimised defocus values. Our simulations indicate that relatively moderate dynamic range (approximately 200 to 1 ) is required for these measurements. This means that this technique is feasible with astronomical sources and normal astronomical receivers.

The technique can only recover large-scale structure of the surface and probably is not suitable for setting individual panels. The possibility, of using astronomical sources and receivers, however, gives several advantages:

1. Measurements of the surface can be performed at a wide range of elevations.

2. The composite phase response of the entire astronomical observing system can be determined, i.e. the effects of deformations of the primary and secondary reflectors and the response of the feed are all included.

3. No investment in hardware or physical changes to the telescope are required. 

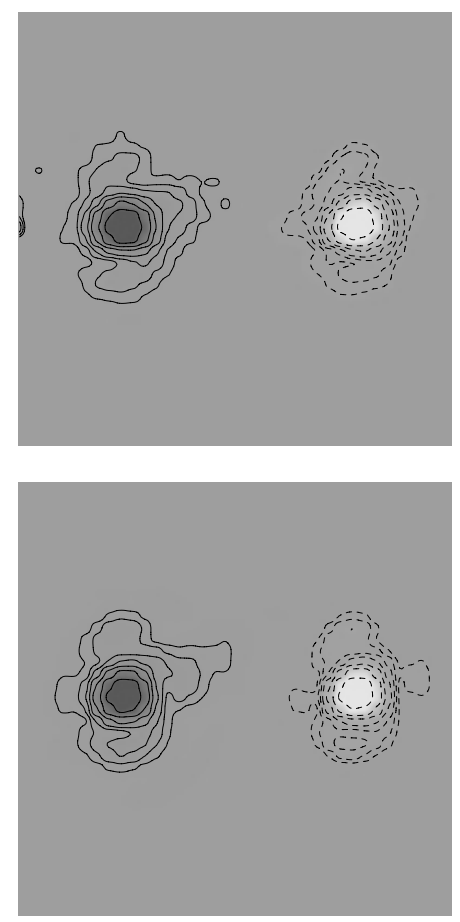
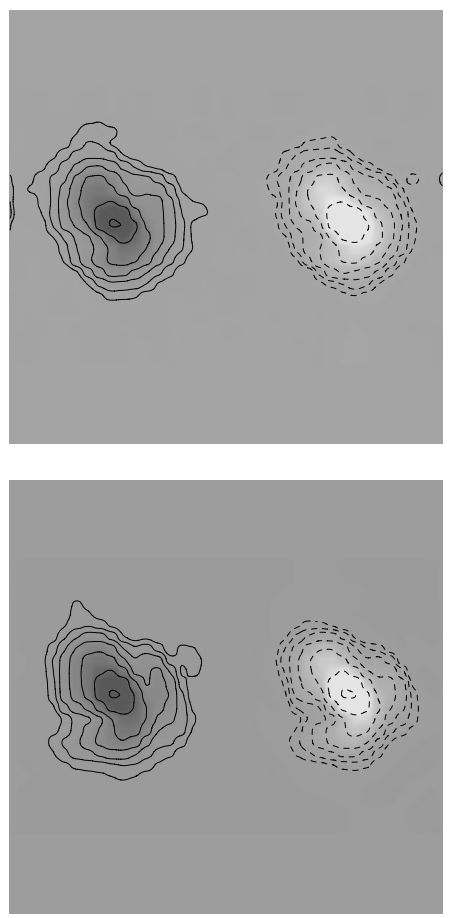
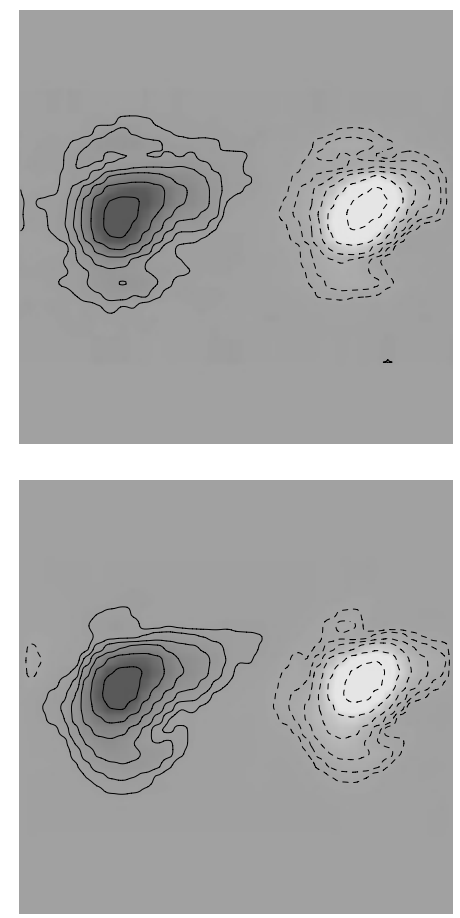

Fig. 4. Comparison of observed in- and out-of-focus beam maps (top row, left to right: in-focus, $+2 \mathrm{~mm}$ and $-2 \mathrm{~mm}$ out-of-focus) and best-fitting model beam maps (using up to 6th order Zernike polynomials, bottom row, same order as top row). Contours are at intervals $2^{-n}$ (thus, the lowest contours are at $-21 \mathrm{~dB}$ in the two leftmost maps and at $-18 \mathrm{~dB}$ in the rightmost map which has one contour less). It is seen that the action of the chopping secondary, followed by synchronous detection, is to produce two beams - one positive and one negative.

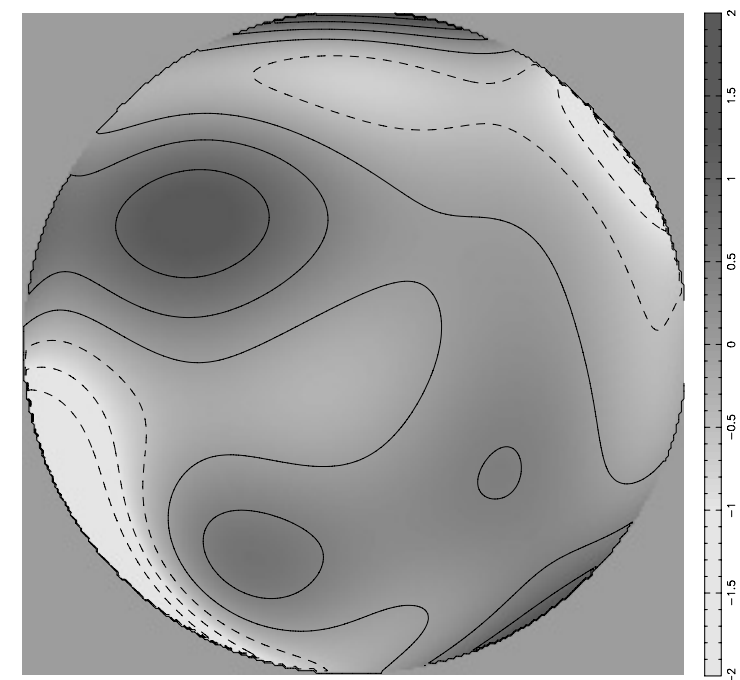

Fig. 5. The inferred aperture-plane phase distribution (in units of radians of phase) from the best-fitting model (using up to 6th order Zernike polynomials) to data shown in Fig. 4. Contours are at fixed intervals of 0.5 radians (corresponding to $51 \mu \mathrm{m}$ ). Positive values represent bumps while negative values represent dips on the surface of the dish.

The combination of first two points above means that the technique should be highly suitable for measurement of gravitation deformations of the telescope surface.

Our simulations and current experience have indicated some drawbacks. One of these is the effect of typically highly tapered astronomical feeds: this significantly reduces the constraints on the outer parts of the dish, although, equivalently, these outer parts contribute less to the effective collecting area of the telescope and therefore do not need to be determined as accurately. The second is that pointing/tracking errors can significantly degrade the accuracy of the technique.

Acknowledgements. We would like to thank Anthony Lasenby for his suggestions which contributed to several aspects of the development of this technique. The James Clerk Maxwell Telescope is operated by The Joint Astronomy Centre on behalf of the Particle Physics and Astronomy Research Council of the United Kingdom, the Netherlands Organisation for Scientific Research, and the National Research Council of Canada.

\section{References}

Anderson, A. P., \& Sali, S. 1985, IEEE Proc., 132, 291

Born, \& Wolf 1970, Principles of Optics (Oxford: Pergamon)

Fuhr, W., Stagulin, J., Schulz, A., et al. 1993, A\&A, 274, 975

Meyerdierks, et al. 1997, Public Domain Algorithms Programmer's Manual, 4th edn., Starlink/CCLRC/Rutherford Appleton Laboratory/PPARC, http://star-www.rl.ac.uk/star/docs/sun194.htx/sun194.html

Morris, D. 1985, IEEE Transactions on Antennas and Propagation, AP-33, 74 Morris, D., Baars, J. W. M., Hein, H., Steppe, H., \& Thum, C. 1988, A\&A, 203, 399

Nikolic, B., Prestage, R. M., Balser, D. S., Chandler, C. J., \& Hills, R. E. 2007, A\&A, 465, 685

Ruze, J. 1966, Proc. IEEE, 54, 663

Scott, P. F., \& Ryle, M. 1977, MNRAS, 178, 539 